\title{
Role of Incentives in Long-term Nutritional and Growth Studies in Children
}

\author{
${ }^{*}$ Nataša Fidler Mis, ${ }^{\dagger}$ Kathy Kennedy, ${ }^{\dagger}$ Mary Fewtrell, ${ }^{\ddagger}$ Cristina Campoy, and ${ }^{\S}$ Berthold Koletzko, \\ for the Working Group on Early Nutrition Research, European Society for Paediatric \\ Gastroenterology, Hepatology and Nutrition (ESPGHAN)
}

\begin{abstract}
Objectives: Available published advice on use of incentives is limited and generally refers to short-term studies without longer follow-up, predominantly conducted in developed countries. We aim to summarize published information related to the use of incentives in long-term nutrition studies involving infants, children, and adolescents and the views of researchers in the field, to provide guidance on acceptable incentives. We conducted a literature review and a short online survey of researchers regarding their opinions on the use of incentives in paediatric long-term (follow-up) clinical studies. Responses from 38 researchers from 14 different countries indicated that $41 \%$ had used incentives to increase participation and $29 \%$ to $73 \%$, depending on child's age and type of procedure, thought incentives may be used to increase compliance with follow-up visits. A small number of respondents thought incentives would not be approved by national ethics boards. Based on the literature review and the survey results, and European Society for Paediatric Gastroenterology, Hepatology and Nutrition working group concluded that incentives for children and adolescents up to the value of 30 Euros, based on average EU income levels, may be offered as cash, vouchers, or age-appropriate gifts or toys, in addition to reimbursing expenses. Additional incentives may be offered if a study includes more burdening procedures, techniques that may appear frightening for younger children, or requires sustained participation (eg, dietary diaries or activity monitoring). There was agreement that it is preferable to give toys or gifts rather than money to younger children.
\end{abstract}

Key Words: infants, long-term nutrition projects, long-term paediatric projects, monetary, nonmonetary

(JPGN 2018;67: 767-772)

Received June 17, 2018; accepted August 27, 2018.

From the *Department of Gastroenterology, Hepatology and Nutrition, University Children's Hospital, University Medical Centre Ljubljana, Ljubljana, Slovenia, the †Childhood Nutrition Research Centre, UCL GOS Institute of Child Health, London, UK, the $\ddagger$ Department of Paediatrics, University of Granada, Granada, Spain, and the \$Division of Metabolic and Nutritional Medicine, LMU-Ludwig-Maximilians-Universität Munich, Dr. von Hauner Children's Hospital, University of Munich Medical Center, Munich, Germany.

Address correspondence and reprint requests to Berthold Koletzko, MD, $\mathrm{PhD}$ Dres.h.c, Professor of Paediatrics, LMU-Ludwig-Maximilians-Universität München, Dr. von Hauner Children's Hospital, Lindwurmstr. 4, 80337 München, Germany (e-mail: office.koletzko@med.lmu.de).

Supplemental digital content is available for this article. Direct URL citations appear in the printed text, and links to the digital files are provided in the HTML text of this article on the journal's Web site (www.jpgn.org).

N.F.M. acknowledges support of the Slovenian Research Agency (P3-0395: Nutrition and Public Health; L3-8213, L3-7538), European Food Safety Authority (EU Menu), and University Medical Centre Ljubljana, Slovenia. The work of C.C. is financially supported in part by the Commission of the European Communities Projects Early Nutrition (FP7-289346), MyNewGut (FP7 613979), and DynaHEALTH (H2020-633595). Additional support has been received from Spanish Ministry of Economy and Competitiveness SFA2015-69265-C2-1-R and BFU2012-40254-C03-01.

\section{What Is Known}

- Advice on using incentives in long-term paediatric nutrition studies is lacking.

\section{What Is New}

- We performed a literature review on acceptable incentives in such studies and a survey among researchers in the field, and developed consensus recommendations of a European Society for Paediatric Gastroenterology, Hepatology and Nutrition working group.

- Incentives with a value up to $30 €$, based on average EU income levels, are considered appropriate for participating children and adolescents.

- Incentives with a higher value could be offered with more burdening procedures, techniques that can appear frightening to children, or data collection that requires sustained participation (eg, detailed dietary protocols, activity monitoring).
M.F. is supported by the Great Ormond Street Hospital Children's Charity. The work of B.K. is financially supported in part by the Commission of the European Communities, Projects Early Nutrition (FP7-289346), DynaHEALTH (H2020-633595) and LIFECYCLE (H2020SC1-2016-RTD), the European Research Council Advanced Grant META-GROWTH (ERC-2012-AdG 322605), the Erasmus Plus programmes "Early Nutrition eAcademy Southeast Asia-573651-EPP-12016-1-DE-EPPKA2-CBHE-JP" and "Early Nutrition eAcademy Southeast Asia-573651-EPP-1-2016-1-DE-EPPKA2-CBHE-JP" and " Capacity Building to Improve Early Nutrition and Health in South Africa598488-EPP-1-2018-1-DE-EPPKA2-CBHE-JP," and the EU Interreg Programme "Focus in CD - CE111." Additional support has been received from the German Ministry of Education and Research, Berlin (Grant Nr. 01 GI 0825), the German Research Council (Ko912/12-1), and the University of Munich Innovation Initiative.

Although this paper is produced by the ESPGHAN Working Group on Early Nutrition Research, it does not necessarily represent ESPGHAN policy and is not endorsed by ESPGHAN.

The authors report no conflicts of interest.

Copyright 102018 by European Society for Pediatric Gastroenterology, Hepatology, and Nutrition and North American Society for Pediatric Gastroenterology, Hepatology, and Nutrition

DOI: 10.1097/MPG.0000000000002143 
A

n "incentive" is "something that motivates or encourages someone to do something', (1). Incentives may motivate a child or parents to participate in research that otherwise would not interest them as much and may help increase research participation at initial recruitment and follow-up visits. How subjects feel about their study experience will influence whether they participate in follow-up studies.

Potential research subjects and their parents should be aware about potential incentives when deciding about study participation. Incentives are offered in addition to reimbursing costs, for example, travel costs. Incentives may be provided as money, gifts, or services (eg, receiving additional care or test results) (2).

The term "incentive" is rarely defined precisely in the paediatric literature. Incentives should not employ "coercion" (ie, intentionally imposing one person's will on another person through the threat of harm or withdrawal of normal privileges, eg, access to healthcare). Incentives should also not appear to be "an inducement,' that is, provide a benefit clearly in excess of the value of study participation, which could appear as "too good to miss", for families.

Here we provide a narrative review of published information relevant to the using incentives in long-term paediatric nutrition trials, report views of researchers in this field, and share recommendations.

\section{METHODS}

We performed a literature search in PubMed, EMBASE, and the Cochrane Central Register of Controlled Trials CENTRAL to identify publications in English relevant to our questions. We searched for the term "incentives" combined with "infant," "child" or "adolescent" and "randomized controlled trial," "clinical trial," "cohort study," "cross sectional study," "observational study," "systematic review," "meta-analysis," "consensus statement," or "guideline." Reference lists of relevant articles were also searched.

We also performed a short online survey with researchers identified as being experienced in paediatric nutrition studies and invited 134 researchers identified by co-authors of this article to complete a short online questionnaire using Survey Monkey (www.surveymonkey.de; SurveyMonkey Europe UC, Dublin, Ireland). The questionnaire was emailed in April $2016(n=49)$ and December $2016(n=85)$. Respondents were asked to consider incentives as "things that children and/or their parents are aware of before they take part in the study and which are designed to motivate or encourage subjects to take part" either as nonmonetary gifts, for example, toys, stationery, cinema tickets, t-shirts, or as monetary incentives. The questionnaire is provided in Appendix 1 (Supplemental Digital Content, http://links.lww.com/MPG/B479).

\section{RESULTS}

\section{Why Use Incentives in Paediatric Research?}

Our review of the published literature confirmed a lack of published information on the use of incentives in long-term paediatric nutrition trials. The reviewed literature also revealed that using incentives in general paediatric research is far from straightforward and clear guidance is sparse; some guidelines refer to the use or prohibition of incentives for recruiting subjects into studies rather than for maintaining cohort participation. Incentives may compensate for lost parental income during a study visit; cash over and above the out of pocket expenses; or film/sporting event tickets, gifts, and toys, entry into a lottery. Further incentives may include easy access to additional clinical services or assessments such as IQ tests, results of blood tests, or dual-energy x-ray absorptiometry (DXA), physical activity monitoring, evaluation of sleep quality, and duration or magnetic resonance imaging (MRI) scans. Whether incentives can enhance cohort retention is largely unknown. Rice and Broome (3) suggest that using incentives may maximize participation, whereas minimizing attrition over time, possibly by increasing feelings of goodwill to researchers, without recruiting a biased sample. They stress that longitudinal studies are more likely than cross-sectional studies to include incentives; longitudinal studies require extended participation by children and parents, presenting a greater burden; thus, incentives are more crucial for recruiting and retaining participants than in studies with limited time commitments.

\section{Current Guidelines and Regulations}

Table 1 summarizes published current guidance, which generally refers to initial recruitment rather than subsequent follow-up. Guidance from the World Medical Association (4) makes no specific provision for the recruitment or follow-up of children in clinical research; regarding incentives it states: "the protocol should include information regarding funding, sponsors, institutional affiliations, potential conflicts of interest, incentives for subjects and provisions for treating and/or compensating subjects who are harmed as a consequence of participation in the research study."'

The European Medicines Agency in 2006 and 2002 (5) conveys similar concepts. It states that institutional review boards/Independent Ethics Committees should review all documents that discuss payments and compensation available to subjects (3.1.2), review both the amount and method of payment to subjects to ensure that neither is seen as coercion or undue influence on the trial subjects. Payments to a subject should be prorated and not wholly contingent on completion of the trial by the subject (3.1.8), and ensure that information regarding payment to subjects, including the methods, amounts, and schedule of payment to trial subjects, is detailed in written information provided to subjects. How payments will be prorated should be specified at recruitment (3.1.9) $(5,6)$.

Regulations of the European Parliament and Council relate only to Clinical Trials on Medicinal Products and not to nutritional interventions (7). They are stricter than those of World Medical Association and European Medicines Agency and state regarding minors: "No incentives or financial inducements are to be given to the subject or his legally designated representative except for the compensation for expenses and loss of earnings directly related to participation in the clinical trial" (32:1(d) (7).

Similarly, the UK Medicines for Human Use Clinical Trials UK recommends "No incentives or financial inducements are given to the minor and to a person with parental responsibility for that minor (except for the provision for compensation in the event of injury or loss)", (8).

In the United States, Devine et al (9) reported that subjects who were likely to conceal or fabricate information to participate in a study also had higher expectations for the value for reimbursement. Rice and Broome (3) published recommendations on the use of incentives for children participating in research in the United States:

1. The incentives should be age appropriate. Younger children are generally pleased with an incentive of comparatively lesser value, whereas adolescents prefer cash or gift cards.

2. Monetary incentives for a child should be based on wage payment model in which the time invested by child or adolescent participants and their parents would be valued relative to payment or a work done and separate from incentives offered to parents. 


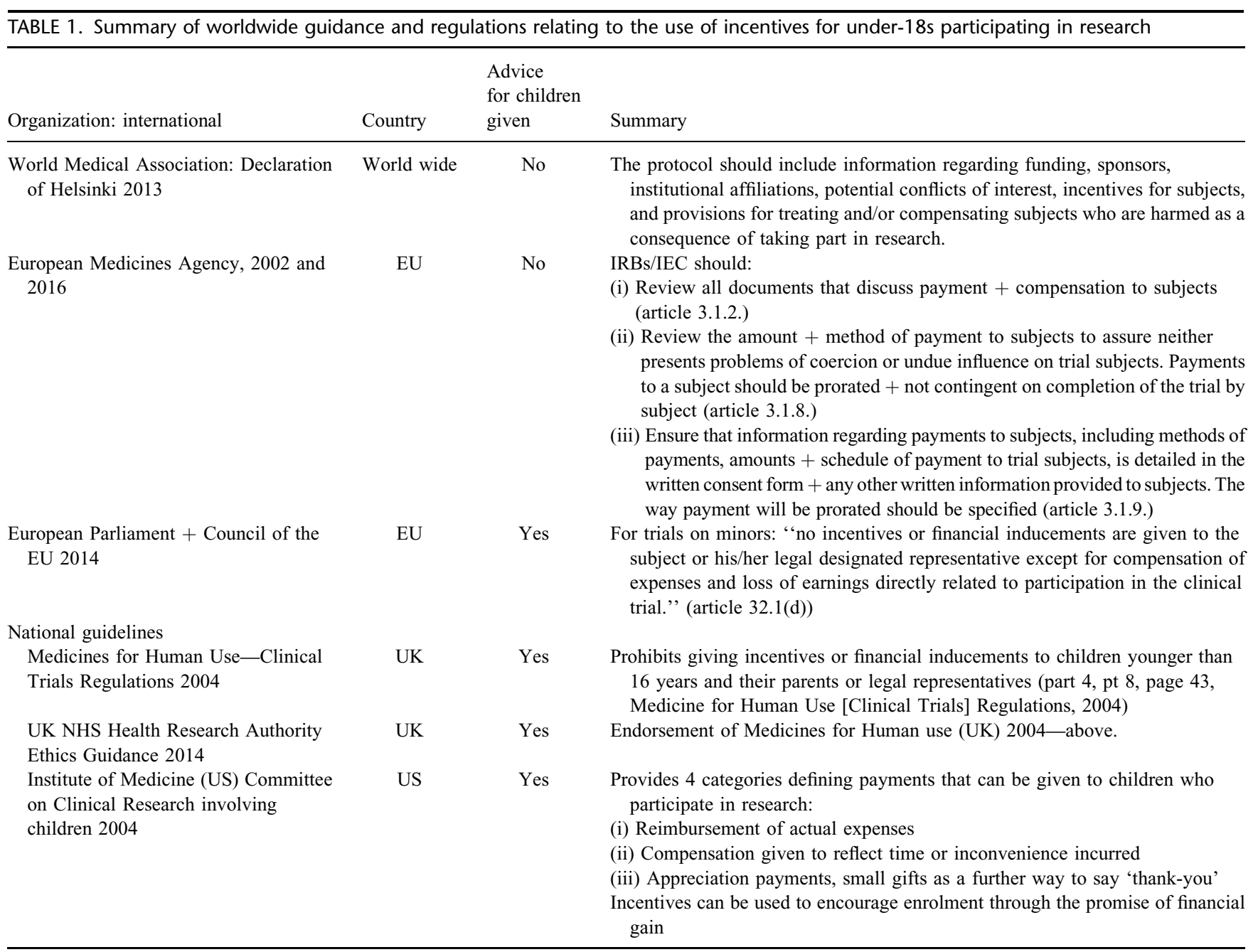

NHS $=$ National Health Service

3. If data collection requires additional burden, such as invasiveness or discomfort, and it is nontherapeutic, the child's monetary or nonmonetary incentive should be increased by at least one third.

4. If the child is the research participant, and the parent is not also a participant, parents should be reimbursed according to the reimbursement model, for expenses only.

5. If a parent and child disagree about whether to participate in a study, the researcher should carefully consider whether the incentives offered might be considered coercive.

The 2014 guidance from the UK National Health Service Health Research Authority (10) endorsed the 2004 UK Medicines for the Human Use Regulations (8) and recommended the use of the previous 2000 Royal College of Paediatrics and Child Health guidance (11) stating researchers should "offer families no financial inducement, although expenses should be paid." In 2014 the UK National Health Service Health Research Authority, however, suggested that for studies other than trials on investigational medicinal products incentives do not need to be prohibited, incentives may well work with older children in much the same way as with adults but younger children may more easily be swayed by gifts or incentives, and vouchers are preferred to cash and should go to the child or directly benefit the child.

The updated UK Royal College of Paediatrics and Child Health guidance on using incentives for children in clinical research (12) considers paid monetary incentives acceptable and defines such payments in 4 categories, based on classifications by Wendler et al (13) of the US National Institute of Health:

1. Reimbursement: payment of reasonable expenses incurred for study participation, for example, travel expenses.

2. Compensation payments: additional monetary rewards for participants for the time, inconvenience, and effort involved in participation.

3. Appreciation payments: a small token given after completion of the study to recognize the voluntary contribution made by the subject.

4. Incentive payments: designed to encourage enrolment through the promise of financial gain.

Concern is expressed that some parents may be unduly influenced to allow their children to participate in research. If this is considered possible, a noncash gift given directly to the child may 
be more appropriate; these are also considered more appropriate for small children who are yet to understand the value of money. Incentive payments may also be used to encourage participants to return data at study end, for example, questionnaires, biological samples (urine, saliva), or tracking device.

Incentives such as taking part in a cash prize draw, often used in adult studies, would generally not be suitable for research with young children as the concept may be lost on them. In adolescents, Seymour (14) used a prize draw with a $25 \%$ chance to win a voucher (20 US \$) successfully and reported a positive effect on recruitment.

Researchers may also consider using "appreciation" payments or gifts to enhance goodwill with their subjects. These should be directed to, and appropriate for, participants rather than responsible adults. In studies with several stages, appreciation gifts may be prorated at each stage. Although appreciation gifts are given at the end of participation and not mentioned at recruitment, they may act as an incentive for future follow-up stages of a study as they can enhance the subject's positive experience regarding study participation.

Comments on use of incentives in research in low-income countries are provided in the supplementary material (Supplemental Digital Content, http://links.lww.com/MPG/B479).

\section{Incentives in Trials on Human Milk Substitutes}

The promotion and protection and support of breast-feeding is paramount for researchers in the field of infant nutrition $(15,16)$. Nevertheless, to evaluate the suitability and safety of human milk substitute (HMS), double-blind randomized controlled trials (RCTs) are required. RCTs on modified HMS that are not approved for commercial use need to provide such HMS for free for the intervention period, similar to drug trials. Recruitment staff should only approach parents who have unequivocally decided to formula feed (usually not within the first days postpartum) for discussing study participation (17). Conditions of studies performed in breastfeeding women and infants, in particular recruitment strategies and interaction with parents and health care professionals, should ensure no interference with breast-feeding (18). For the complementary feeding period, food products that are part of the protocol even beyond the experimental period may be offered; for example, cereals, to reduce dietary variability. Researchers recruiting infants into feeding studies should show complete transparency regarding their recruitment methods. The provision of free trial HMS should not serve to persuade parents to participate, and one could argue that it should not be mentioned until the consent has given, but others would contend that it is unethical not to tell parents that they are asked to use study products and they should know what they will be asked to feed their infant for how long. HMS is normally only provided during the intervention phase of a trial and not considered an incentive for participation in longer-term follow-up.

\section{Views of Researchers Regarding Incentives in Long-term Follow-up Studies}

Thirty-eight of 134 researchers $(28.4 \%$ of those invited for survey participation) from 12 European countries responded. Approximately 58\% (22/38) had personal experience in long-term paediatric studies; these included collecting anthropometric data, ultrasound measurements, and activity monitoring. Results showed that $41 \%(9 / 22)$ of researchers with such experience routinely used incentives for different data collection techniques, or had provided gift vouchers, small toys, and breast-feeding equipment (Table 2). For children subjected to venous blood sampling ages younger than 5,6 to 11 , and older than 12 years, respectively, $68 \%, 71 \%$, and $70 \%$ of respondents thought offering incentives was appropriate, with similar results for capillary blood samples. More than $67 \%$ thought that incentives were suitable for data collection techniques that require several days, for example, activity monitoring or collecting dietary data, with similar results for imaging procedures, for example, MRI or DXA. Less than 50\% thought that incentives were necessary for data collection such as anthropometry and saliva collection. Urine collection for children older than 5 years was thought not to require incentives by $50 \%$ of researchers. Most researchers thought an incentive valued $<30 €$ was suitable; fewer than $5 \%$ thought incentives should be worth $>50 €$, and a similarly small number considered a donation to a charity as an incentive. In free text comments, $58 \%$ (22/38) suggested incentives may have a role in long-term research with children, whereas only $3(8 \%)$ felt incentives should not be used. Key opinions are collated in Supplementary Appendix 2 (Supplemental Digital Content, http:// links.lww.com/MPG/B479).

\section{DISCUSSION}

Published guidance on using incentives in paediatric research is focussed on drug trials and the recruitment and intervention phases, whereas there is little guidance on nutrition studies and long-term follow-up. Long-term studies of infants and children are important since they can provide insights into the efficacy and safety of dietary and other interventions in early life; both RCTs and prospective cohort studies are widely used to understand the longterm effects of early exposures, test hypotheses in developmental programming and identify causal pathways $(19,20)$. In clinical drug trials of children (and adults) affected by a disease, the opportunity to obtain improved therapies, and additional clinical care during study visits may provide a strong incentive for participation in both clinical trials and subsequent follow-up visits. In contrast, nutritional studies often enrol healthy infants and children who have no disease-related motivation for participation beyond the initial data collection phase. Long-term follow-up studies often carry perceived burdens of time and inconvenience, which may be off-putting for the subject or their families. Attrition or uncontrolled loss of subjects in long-term follow-up studies is often high and results in statistical concerns including introduction of bias, generalizability, and loss of power (21). Despite efforts to minimize attrition, present experience suggests that it is to some extent inevitable in long-term follow-up studies. The use of appropriate incentives may provide opportunities to attenuate the degree of attrition.

Our survey of international researchers conducting paediatric nutrition trials showed that most had supported incentives with more burdening data collection (eg, blood samples), data collection that requires sustained participation (eg, dietary data or activity monitoring), or techniques that may frighten younger children (eg, MRI scans). They considered incentives less likely to be justified with techniques that are quick to perform (anthropometry) and noninvasive (saliva, urine collection). Only a very small minority of researchers $(<1 \%)$ do not consider incentives as appropriate or useful.

A limitation of our survey is the small number of researchers included. To our knowledge no surveys of children or their caregivers were conducted, although attitudes of adults were surveyed in the USA $(22,23)$. No randomized trials on effects of incentives at recruitment of study participants in children are published. In adults, Jennings et al (24) randomized subjects to receive study invitation letters offering incentives or not and found a $£ 100$ cash incentive to induce a small but significant increase in recruitment, although this did not attract elderly or more socially deprived participants.

Future guidance is needed to ascertain when incentives are acceptable, for example, if more onerous data collection methods are employed and if possible, to identify appropriate values of 

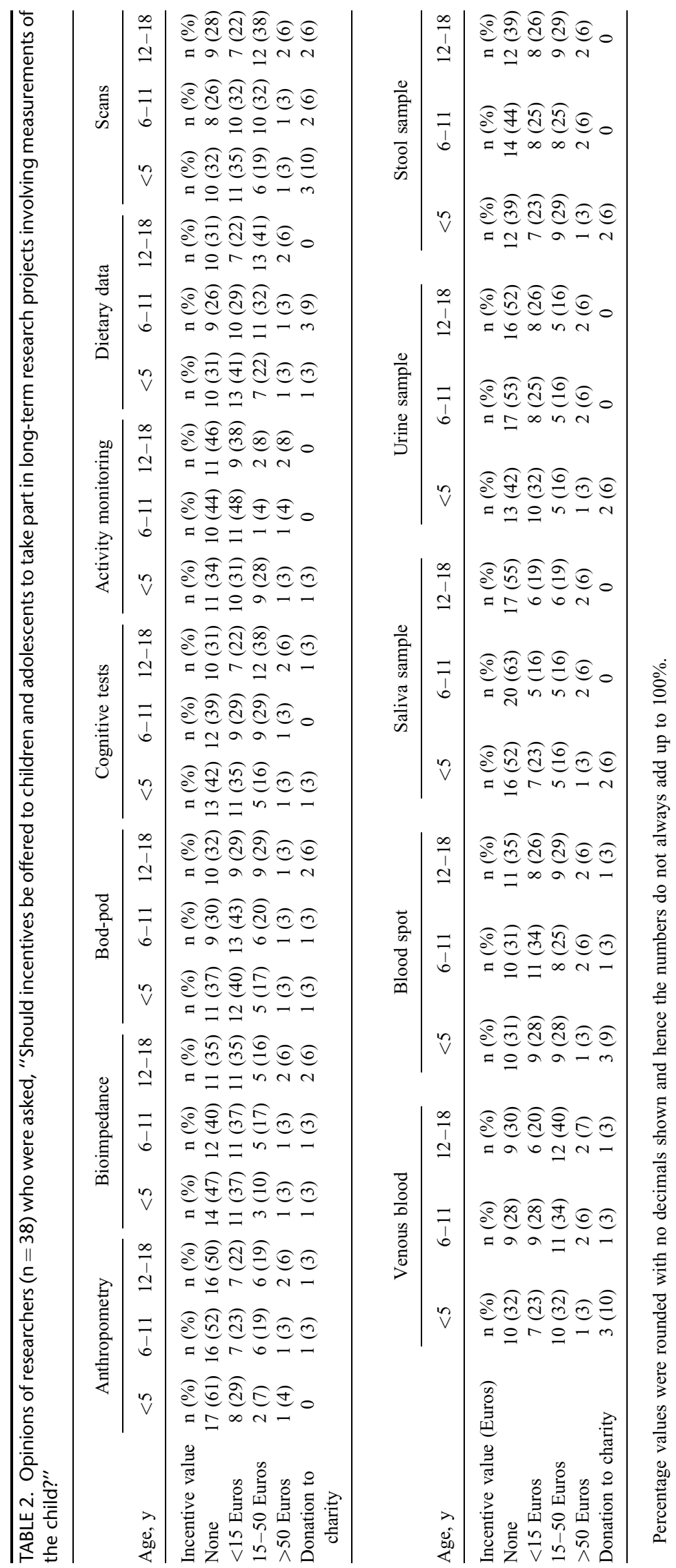
incentives. We consider incentives to likely play an important role in cohort maintenance, particularly when researchers require subjects and their families to take part in several phases of data collection over many years. A positive experience for the subject and their families can conceivably act as "incentives", for future participation; such an experience could be due to many factors but include friendly, competent staff, appointments carried out at convenient times and places, good time keeping, provision of interesting, or helpful information that they would not normally receive (eg, results from an IQ test or a DXA scan image).

\section{Consensus Recommendations}

1. The European Society for Paediatric Gastroenterology, Hepatology and Nutrition Working Group on Early Nutrition Research gathered at the European Society for Paediatric Gastroenterology, Hepatology and Nutrition annual congress 2017 and unanimously agreed:

2. Incentives with a value up to $30 €$ (in EU countries or with comparable cost of living) may be provided as monetary incentives or vouchers, toys, cinema tickets, t-shirts, etc.

3. For younger children providing toys or gifts rather than money is considered preferable.

4. Higher values of incentives may be considered in situations with a. More burdening data collection such as blood samples,

b. Data collection requiring sustained participation (eg, dietary data, activity monitoring), or

c. Techniques that appear frightening for younger children (eg, MRI scans).

5. In addition, reimbursement of actual expenses related to the participation in the study and study visits may be offered, including reasonable compensation for loss of work time.

\section{REFERENCES}

1. Oxford-Dictionaries. http://en.oxforddictionaries.com/definition/incentive. Accessed June 16, 2018.

2. Polacsek M, Boardman G, McCann TV. Paying patient and caregiver research participants: putting theory into practice. $J$ Adv Nurs 2017;73:847-56.

3. Rice M, Broome ME. Incentives for children in research. $J$ Nurs Scholarsh 2004;36:167-72.

4. World Medical Association. WMA Declaration of Helsinki. Ethical Principles for Medical Research Involving Human Subjects. Fortaleza; 2013.

5. European Medicines Agency. ICH Topic E6 (R1) Guideline for Good Clinical Practice. London: European Medicines Agency; 2002.

6. European Medicines Agency. Guideline for Good Clinical Practice E6 (R2), Vol. 6. London: European Medicines Agency; 2016.
7. European Parliament and Council of the European Union Regulation (EU) No 536/2014 of the European Parliament and of the Council of 16 April 2014 on clinical trials on medicinal products for human use, and repealing Directive 2001/20/EC. Off J Eur Union 2014 2014:L1-L76.

8. The Medicines for Human Use (Clinical Trials) Regulations. Government of the United Kingdom 2004. http://www.legislation.gov.uk/uksi/ 2004/1031/contents/made.

9. Devine EG, Knapp CM, Sarid-Segal O, et al. Payment expectations for research participation among subjects who tell the truth, subjects who conceal information, and subjects who fabricate information. Contemp Clin Trials 2015;41:55-61.

10. National Health Service Health Research Authority. HRA Ethics Guidance: Payments and Incentives in Research. Volume 1. hra-guidancepayments-incentives-research\%20(3).pdf. Accessed June 16, 2018.

11. McIntosh N, Bates P, Brykczynska G, et al. Guidelines for the ethical conduct of medical research involving children. $R$ Coll Paediatr Child Health 2000;82:177-82.

12. Modi N, Vohra J, Preston J, et al. Guidance on clinical research involving infants, children and young people: an update for researchers and research ethics committees. Arch Dis Child 2014;99:887-91.

13. Wendler D, Rackoff JE, Emanuel EJ, et al. The ethics of paying for children's participation in research. J Pediatr 2002;141:166-71.

14. Seymour-K. Using incentive: encouraging and recognising participation in youth research. Youth Stud Aust 2012;31:51-9.

15. ESPGHAN-Committee-on-Nutrition, Agostoni C, Braegger $\mathrm{C}$, et al. Breast-feeding: a commentary by the ESPGHAN Committee on Nutrition. J Pediatr Gastroenterol Nutr 2009;49:112-25.

16. Prell C, Koletzko B. Breastfeeding and complementary feeding-recommendations on infant nutrition. Dtsch Arztebl Int 2016;113:Error: FPage (435) is higher than LPage (344)!.

17. Koletzko B, Ashwell M, Beck B, et al. Characterisation of infant food modifications in the European Union. Ann Nutr Metab 2002;46:231-42.

18. Koletzko B, Benninga MA, Godfrey KM, et al. Public-private collaboration in clinical research during pregnancy, lactation, and childhood: joint position statement of the Early Nutrition Academy and the European Society for Pediatric Gastroenterology, Hepatology, and Nutrition. J Pediatr Gastroenterol Nutr 2014;58:525-30.

19. Koletzko B, Brands B, Grote V, et al. Long-term health impact of early nutrition: the power of programming. Ann Nutr Metab 2017;70:161-9.

20. Rauschert S, Kirchberg FF, Marchioro L, et al. Early programming of obesity throughout the life course: a metabolomics perspective. Ann Nutr Metab 2017;70:201-9.

21. Fewtrell MS, Domellof M, Hojsak I, et al. Attrition in long-term nutrition research studies: a commentary by the European Society for Pediatric Gastroenterology, Hepatology, and Nutrition Early Nutrition Research Working Group. J Pediatr Gastroenterol Nutr 2016;62:180-2.

22. Largent EA, Grady C, Miller FG, et al. Money, coercion, and undue inducement: attitudes about payments to research participants. IRB 2012;34:1-8.

23. Czarny MJ, Kass NE, Flexner C, et al. Payment to healthy volunteers in clinical research: the research subject's perspective. Clin Pharmacol Ther 2010;87:286-93.

24. Jennings CG, MacDonald TM, Wei L, et al. Does offering an incentive payment improve recruitment to clinical trials and increase the proportion of socially deprived and elderly participants? Trials 2015;16:80. 